\title{
BMAD. Automatic Detection of Flight Manoeuvres using wavelets
}

\author{
Francisca Coll Herrero \\ Airbus Defence and Space \\ Francisca.Coll@airbus.com
}

\begin{abstract}
:
BMAD is a big data oriented project which aims to automatize most of the flight test analysis process. The idea is to classify and identify automatically all the manoeuvres of ADS (Airbus Defence and Space) aircrafts fleet.

Up to now manoeuvres are selected interactively using plotting tools and in some cases these time slices are not stored in a data base.

The project consists in the automatic identification of maneuvers, the storage of their representative data in a database, and the exploitation of the data in a web service.

This paper will describe the use of wavelets for the detection of typical flight quality manoeuvres applied to real data corresponding to a combat aircraft.
\end{abstract}

Key words: BMAD: Bigdata Manoeuvre Automatic Detection, SQL: Structured Query Language, NoSQL: Not only SQL, Ftnet: Flight Test Net, CSV: Comma separate values, HDF5 : Hierarchical Data Format, JSON : JavaScript Object Notation.

\section{Introduction}

BMAD starts from the need of automatizing Flight Test data analysis chain.

This process covers: data acquisition and processing, time slice detection and manoeuvre classification, manoeuver's calculus and data storage, flight test report

generation and data exploitation: web services $\&$ dashboards.

The first step is to classify and identify automatically all the manoeuvres of ADS (Airbus Defence and Space) aircrafts fleet.

Each manoeuvre corresponds to a time slice and it is identified by an initial and final time. There are also some calculations associated to each manoeuvres like maximum values, minimum values, mean, standard deviation, even complex calculus like parameter identification .In most cases, the manoeuvres are selected by the analyst using interactive plotting tools.
Some manoeuvres like take off, landing or stabilizations can be easily identified using simple rules, for these cases we have an inhouse developed tool called Event Detector. Event Detector is a program written in Java in which you can define with a rule the initial time and the final time of a manoeuvre.

But for other kind of manoeuvres it is necessary to use other techniques of identification using complex patterns.

Different technologies have been tested like neural network and wavelets the latter obtaining better results.

Once the manoeuvres are identified, in-house software, called JProcessor, is used for automatically performing desired data reduction.

The project consists in the automatic identification of manoeuvres, storing the times slices, calculations, and even the complete manoeuvre in a NoSQL (not only SQL) data base and the exploitation the data in a web service. 
This paper will focus on the use of wavelets for the detection of typical Flight Test manoeuvres following these steps:

a) Define the pattern signal corresponding to the manoeuvre.

b) Obtain the best mother wavelet adapted to this pattern.

c) Find the wavelet in the time series by translation and dilations of the mother wavelet using the Scalogram.

\section{BMAD workflow}

In the Figure 1 it is shown the diagram of the BMAD project:

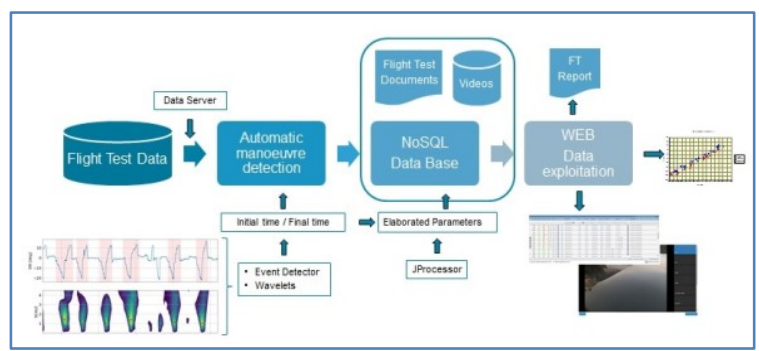

Figure 1.BMAD workflow

a) Access to the flight test data using data server.

b) Automatic manoeuvre determination using Event Detector and/or Wavelets.

c) Automatic elaborated parameter calculations (JProcessor).

d) Store desired information in a NoSQL data base.

e) The server includes in addition to NoSQL data base, all flight information: documents, videos and relational data base.

f) Finally, via web service (FTNet), you can access to all flights information, status of the program, plotting, streaming video service and even an automatic generation of Flight Test Reports.

\section{Data Reduction, Parallel Processing}

BMAD adopts many Big Data concepts during the data reduction phase: a) Analysis focuses on multiples manoeuvres, different flights and several prototypes.

b) Paralleling processing is used for improved performance, relying on MapReduce concept.

c) Increasing the number of machines improves the speed of the analysis process. Tasks are assigned using a task scheduler (daemon) which controls the work flow of the phase.

d) Queuing is used to minimize idle times and improve data reduction efficiency.

Data reduction and calculus are performed by JProcessor. This application is an in-house developed tool coded in Java. This software is plug-in based, meaning new calculus functions can be added without modifying its structure.

JProcessor reads data, builds a data processing tree and calls elaboration functions. Finally, when all data is computed, it can write the results to several formats: databases (SQL \& NoSQL), data files (CSV, CDF, HDF5), JSON etc.

\section{Data Exploitation}

Once the process of automatic manoeuvre detection, involved calculus and the storage in the data base is completed, BMAD process includes data exploitation via web -based services.

The web service will contain:

a) General Flight information: date, flight objective, aircraft configuration....

b) Manoeuvres information: initial time, final time, calculus results ...

c) Status of the program : test point key status ...

d) Plotting cross-plots, comparing different flights....

e) Streaming video service.

f) Automatic generation of Flight Test Reports. 


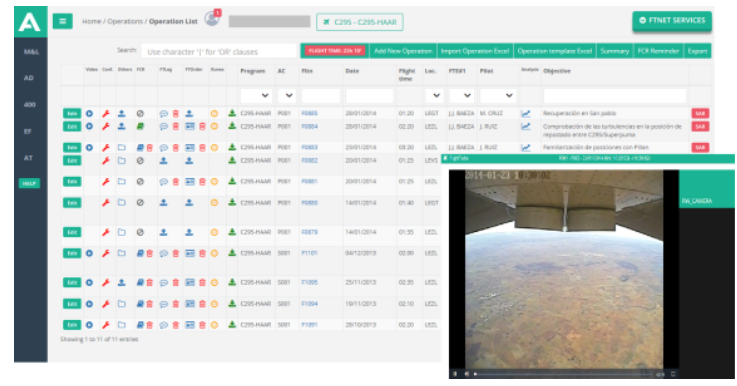

Figure 2. Web service

\section{Analysis Methodology, Why Wavelets}

The objective is the automatic pattern detection in time series.

Typical algorithms used are:

a) Short time Fourier Transform, this analysis has some limitations because it only works in frequency domain.

b) Neural networks, they need a lot of time for the training and results are not good enough.

c) Continuous wavelet transforms. Is similar to the Fourier analysis but it works not only in frequency domain but also in time domain. Fehler! Verweisquelle konnte nicht gefunden werden.

Wavelet analysis expands functions in terms of translations and dilation of a wavelet function called mother wavelet.

A wavelet is a function $\psi \in \mathrm{L}^{2}(\mathrm{R})$ with zero average (i.e. $\int_{R} \psi=0$ ), normalized (i.e. $\|\psi\|=$ 1 ), and centred in $\mathrm{t}=0$.

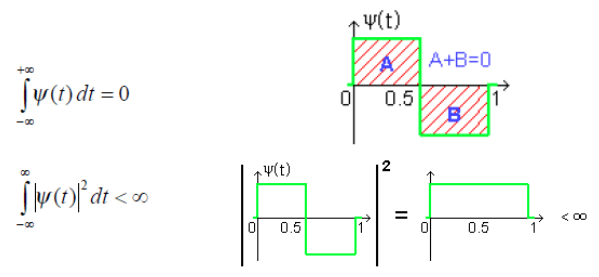

Figure 3. Haar wavelet

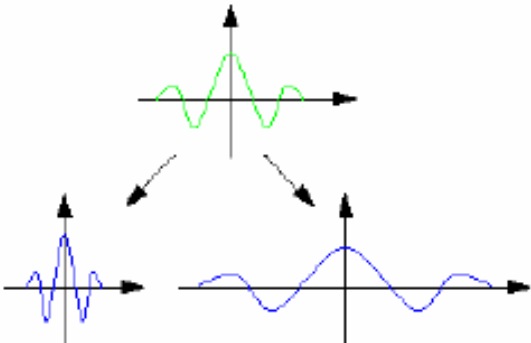

Figure 4. Mother wavelet $\psi$

Scaling $\psi$ by a positive quantity $s$, and translating it by $u \in R$, we define a family of functions obtained from "mother" wavelet $: \psi_{\mathrm{u}, \mathrm{s}}$,

$\boldsymbol{\Psi}_{\mathrm{u}, \mathrm{s}}(\mathrm{t})=\frac{1}{\sqrt{\mathrm{s}}} \boldsymbol{\Psi}\left(\frac{\mathrm{t}-\mathrm{u}}{\mathrm{s}}\right), \quad \mathrm{u} \in \mathbb{R}, \mathrm{s}>\mathbf{0}$

Given $\mathrm{f}$, the continuous wavelet transform (CWT) of $f$ at time $u$ and scale $s$ is defined as:

$W f(u, s)=\int_{-\infty}^{+\infty} f(t) \psi_{u, s}^{*}(t) d t$

\section{Fourier versus wavelets}

Fourier analysis has some limitations because only works in frequency domain. Sometime later, the notion of scale analysis by creating structures that vary in scale was developed

The most interesting difference between these two kinds of transformations is that individual wavelet functions are localized in space. Fourier sine and cosine functions are not. [5]

A wavelet is a mathematical function useful in digital signal processing and image compression. [2],[5]

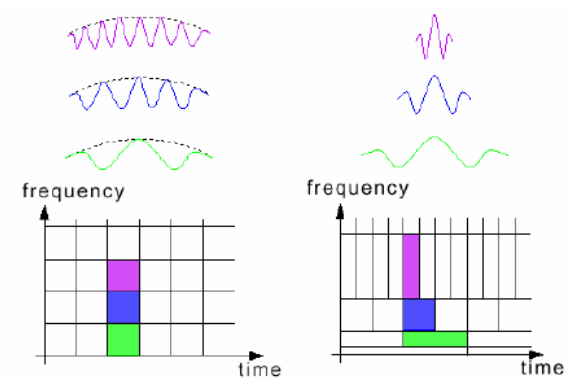

Figure 5. Time-Frequency plane :Fourier transform 


\section{Construction of Wavelets Approaching a Pattern}

The technique described in this paper is based on the correlation of each manoeuvre with a pattern.

The first step is to construct wavelets adaptable, as well as possible, in the least squares sense, to the specific pattern.

But it is no using the typical wavelet library like Haar, b-spline,daubechies ,coiflet or Mexican hat, it is adapting each pattern to a sum of linear combinations of orthogonal polynomial [6].

Take the family of $\mathrm{N}$ functions $\mathrm{F} \in L^{2}(\mathrm{a}, \mathrm{b})$

$$
F=\left\{\rho_{i}\right\}_{i=1}^{N}
$$

We want to construct the approximation of the pattern $=f$ in the least square sense using a linear combination of the $\mathrm{F}$ :

$$
\psi=\sum_{i=1}^{N} \alpha_{i} \rho_{i}
$$

with the condition that:

$$
\sum_{i=1}^{N} \alpha_{i} R_{i}=0
$$

Where:

$$
R_{i}=\int_{a}^{b} \rho_{i}(t) d t
$$

The vector $\left\{\alpha_{i}\right\}_{i=1}^{N}$ and the function $\psi$ are obtained by solving the following linear system where:

$\left(\begin{array}{cc}G & M^{t} \\ M & 0\end{array}\right)\left(\begin{array}{l}\alpha \\ \lambda\end{array}\right)=\left(\begin{array}{l}B \\ 0\end{array}\right)$

Where :

$G_{i j}=\int_{a}^{b} \rho_{i}(t) \rho_{j}(t) d t$

$M_{i=} R_{i}$
$B_{i}=\int_{a}^{b} f(t) \rho_{i}(t) d t$

In numerical calculations, we have a finite sets of values of the function in an interval $[a, b]$ :

$\left\{\left(t_{k}, y_{k}\right)\right\}_{k=1 \ldots N}$ where $a \leq t_{k} \leq b$

and $f\left(t_{k}\right)=y_{k}$

The problem consists in to find

$\alpha=\left\{\alpha_{i}\right\}_{i=1}^{N}$ and $\psi=\sum_{i=1}^{N} \alpha_{i} \rho_{i}$

Such that minimize in least square sense the expression:

$\sum_{1}^{N}\left[\psi\left(t_{k}\right)-y_{k}\right]^{2}$

With the constraint:

$$
\int_{a}^{b} \psi(t) d t=0
$$

The vector $\alpha$ is obtained by solving the equation (7).

\section{Manoeuvre Detection using Wavelets}

The steps used to identify the manoeuvres using wavelets are the followings:

a) Select a pattern of the specific manoeuvre.

b) Obtain the corresponding mother wavelet adapted to this pattern.

c) Find the pattern in the time series by translation and dilations of the mother wavelet function using Scalogram. Ref.3.

The following Figures 4, 5, 6 represent manoeuvre identifications steps applied in a theoretical signal composed by sines:

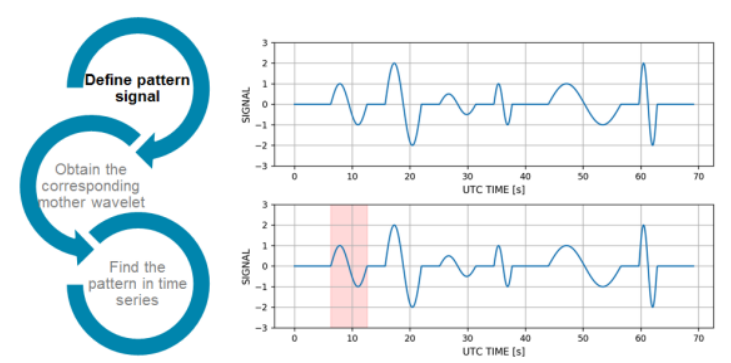

Figure 6.Define pattern signal: SETP1 
In this case the mother wavelet is the sine function:
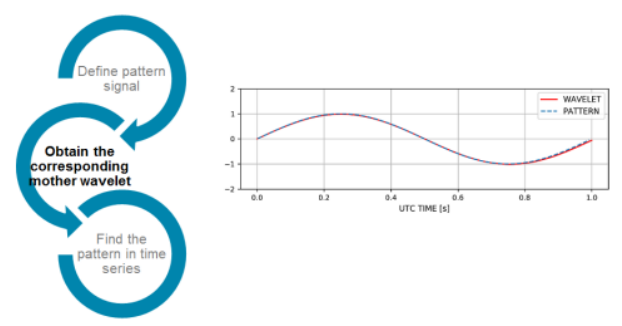

Figure 7.Mother wavelet : STEP2
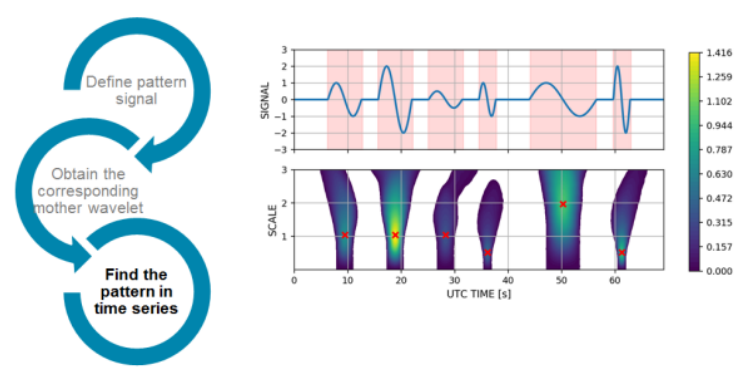

Figure 8.Find pattern in time :STEP 3

\section{Examples}

This section shows cases of the usage of wavelets for the identification of manoeuvres corresponding to a Combat aircraft.

\section{a) Rapid Roll}

The following figures correspond to Flight Quality test on a Combat aircraft which contains a series of Rapid Roll (lateral directional manoeuvre).

- $\quad$ The first step is to select the pattern on the STK_PARAM parameter:

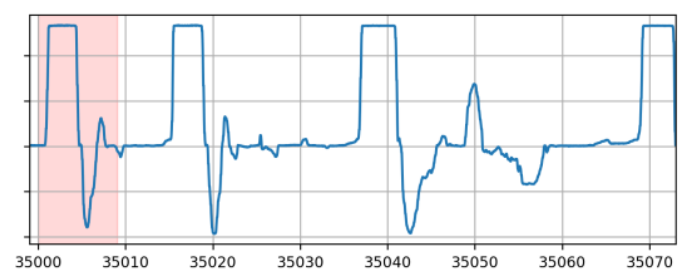

Figure 9.Define pattern

- The second step is to obtain the corresponding mother wavelet:

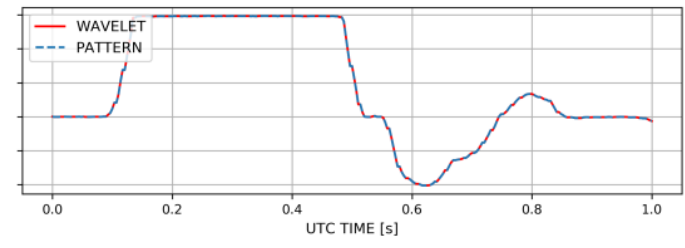

Figure 10.Mother wavelet

- The third step is to find the pattern in to the time series.

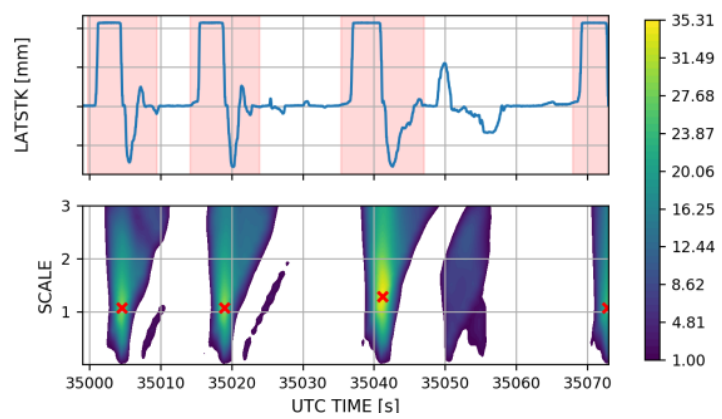

Figure 11. Find pattern in time

b) Lateral directional triplets

The following example will demonstrate that it is possible to use this technique to identified complex manoeuvres like lateral directional triplets that is compound by three simple doublets. The input of this manoeuvre is generated mathematically and applied directly to the Flight Control System (FCS Bias input).

First it will use the $\mathrm{FBI}$ input to define the pattern and second the Rudder parameter, the times slices of each identified manoeuvres will be the same in both cases.

- The first step is to select the pattern on the FBI_PARAM parameter:

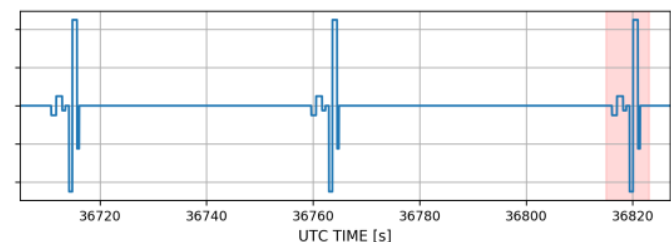

Figure 12.Define pattern

- The second step is to obtain the corresponding mother wavelet: 


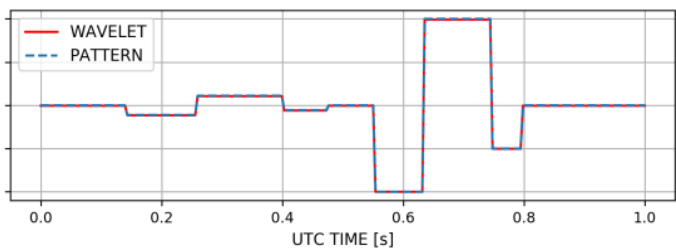

Figure 13.Mother wavelet

- The third step is to find the pattern in to the time series.

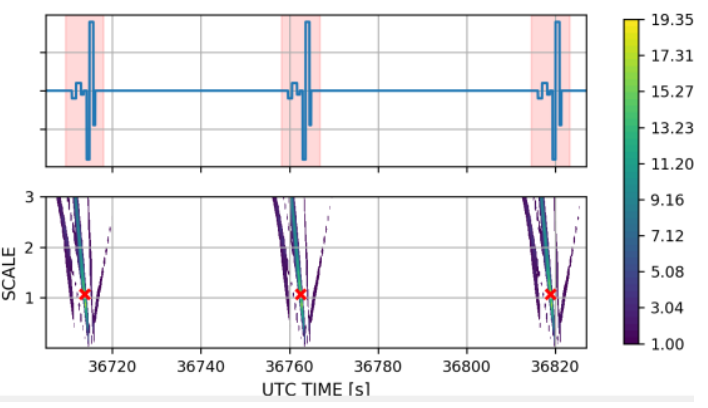

Figure 14. Find the pattern in time

The following example the Rudder parameter is used as a pattern:

- The first step is to select the pattern on the DR parameter:

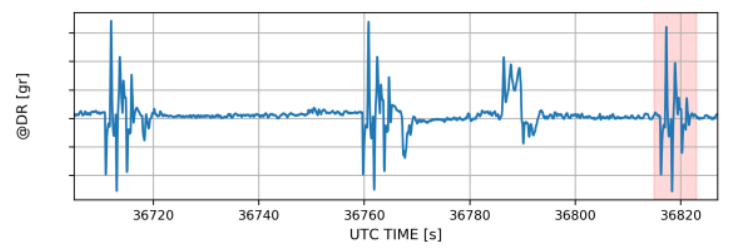

Figure 15.Define the pattern

- The second step is to obtain the corresponding mother wavelet:

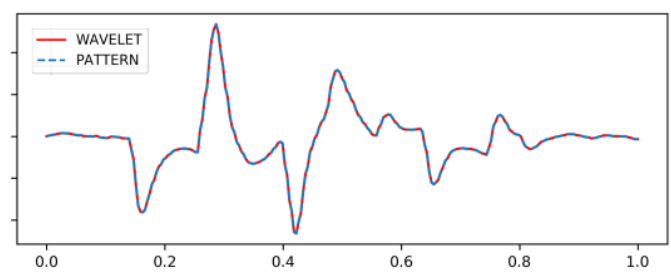

Figure 16.mother wavelet

- The third step is to find the pattern in to the time series.

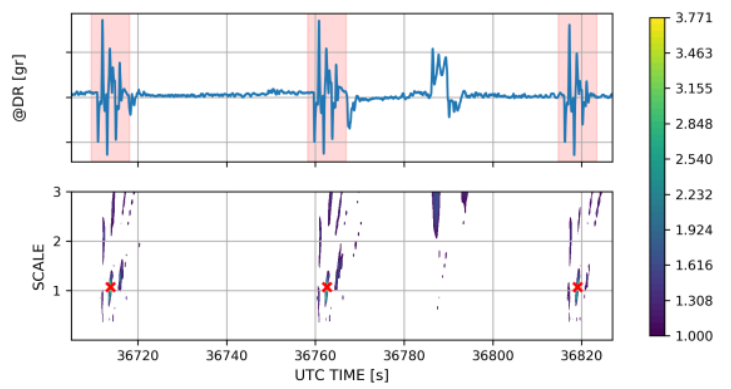

Figure 17. Find the pattern in time

\section{Find Pattern Tool}

The tool to find patterns using wavelets is an in-house software written in Python.

First a library of mother wavelets corresponding to each manoeuvre is generated.

This library is composed by synthetic and real manoeuvres.

Once the library is created, the steps for the automatic manoeuvre detection are the following:

a) Select the fight to find the manoeuvres.

b) Select the initial and final time (by default is applied to the full flight

c) Select the list of flight parameters used to search the pattern.

d) Select the wavelet from de library and the correlation and scale margins.

e) Use the wavelet to find Find all the manoeuvres selected in the flight.

f) Store the time slices corresponding to each manoeuvre in the data base.

The following figure shows the interface of the tool. 


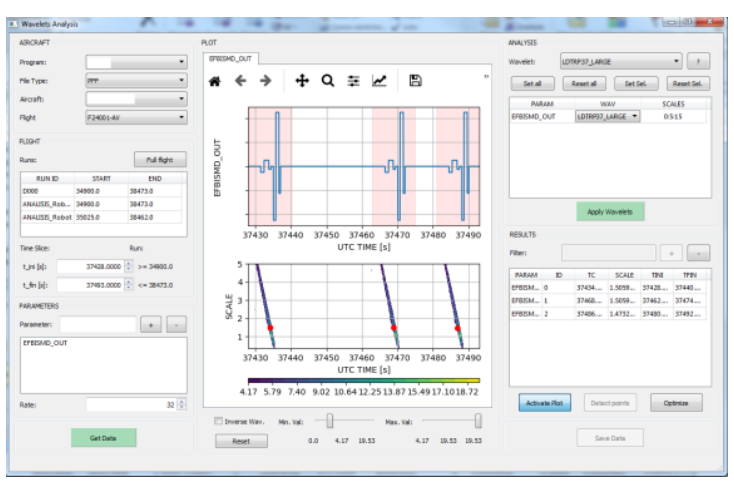

Figure 18. Find Pattern Tool

\section{Conclusions}

Nowadays, the Flight Test analysis process is a mostly manual procedure.

The main idea of BMAD is to improve analysis chain by:

a) Minimizing hand-made processes, prone to human error (machine learning techniques).

b) Automatic error detection through Big Data analysis procedures.

c) Reducing the time need to generate reports and documents.

d) Improving data access throw digitalization and webs tools.

\section{References}

[1] Paola Andrea Quiñones Roa. "TRANSFORMADA WAVELET, LOCALIZACIÓNTIEMPO FRECUENCIA Y ANÁLISIS DE CIRCUITOS RLC “, Universidad Distrital Francisco Jose de Caldas. Facultad tecnológica.

[2] Liesner Acevedo Martinez. "Computación paralela de la transformada Wavelet; Aplicaciones de la transformada wavelete al álgebra lineal numérica ». Departamento de Sistemas informáticos .Universidad Politécnica de Valencia.Mayo 2009

[3] V.J. Bolos,R. Benitez ."The wavelet scalogram in the study of times series". XXIII Congreso de Ecuaciones Diferenciales y Aplicaciones .XIII Congreso de Matematica Aplicada. 2013.

[4] Noelia Rillo Pastor. "Introducción a la teoría de wavelets".Departamento de matemática aplicada y análisis , universidad de Barcelona .2005

[5] Amara Graps. "An indroduction to Wavelets".

[6] Wavelets and theirs applications. Michel Misiti, Yves Misiti, Georges Oppenheim and Jean-Michel Poggi 